\title{
Association of white matter hyperintensities with migraine features and prognosis
}

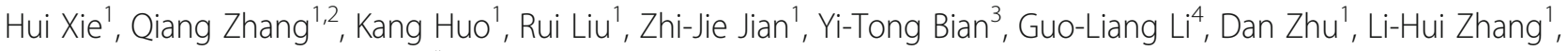 \\ Jian Yang ${ }^{3}$ and Guo-Gang Luo ${ }^{1 *}$
}

\begin{abstract}
Background: White matter hyperintensities (WMHs) are frequently detected in migraine patients. However, their significance and correlation to migraine disease burden remain unclear. This study aims to examine the correlation of WMHs with migraine features and explore the relationship between WMHs and migraine prognosis.

Methods: A total of 69 migraineurs underwent MRI scans to evaluate WMHs. Migraine features were compared between patients with and without WMHs. After an average follow-up period of 3 years, these patients were divided into two groups, according to the reduction of headache frequency: improved and non-improved groups. The percentage and degree of WMHs were compared between these two groups.

Results: A total of 24 patients (34.8\%) had WMHs. Patients with WMHs were significantly older (39.0 \pm 7.9 vs. $30.6 \pm$ 10.4 years, $P<0.001$ ) and had a longer disease duration (median: 180.0 vs. 84.0 months, $P=0.013$ ). Furthermore, 33 patients completed the follow up period (15 patients improved and 18 patients did not improve). Patients in the non-improved group had a higher frequency of WMHs $(55.6 \%$ vs. $13.3 \%, P=0.027)$ and median WMHs score (1.0 vs. $0.0, P=0.030$ ).
\end{abstract}

Conclusions: WMHs can predict unfavorable migraine prognosis. Furthermore, WMHs may have a closer association with age than migraine features.

Keywords: Migraine, White matter hyperintensities, Clinical significance, Prognosis

\section{Background}

Migraine is a chronic debilitating headache characterized by recurrent moderate-to-severe headache attacks and autonomic nervous system related-symptoms [1]. Globally, migraine affects approximately $15 \%$ of the general population, and it preferentially affects females [2]. Migraine can be regarded as a risk factor associated with white matter hyperintensities (WMHs) [3], which are hyper-intense brain lesions in T2-weighted and Fluid-Attenuated Inversion Recovery (FLAIR) images [4]. Accumulating evidence documented the high incidence of WMHs in patients with migraine $[3,5]$. However, the exact correlation between WMHs and the clinical features of migraine remain

\footnotetext{
*Correspondence: Iguogang@163.com

'Department of Neurology, The First Affiliated Hospital of Xi'an Jiaotong

University, No. 277 Yanta West Road, Xi'an 710061, Shaanxi, China

Full list of author information is available at the end of the article
}

unclear. A population-based CAMERA study suggested the increased risk of WMHs in migraine patients with higher attack frequencies ( $\geq 1$ attack per month), compared with patients with lower attack frequencies $(<1$ attack per month) [3]. Trauninger et al. demonstrated that both disease duration and attack frequency were associated with WMHs in migraine patients [6]. On the other hand, Toghae et al. observed an association between WMHs and the age and migraine duration of patients, but not with attack frequency [7]. Recent studies have reported that WMHs are associated with the age of patients, and not with disease burden (disease duration and attack frequency) $[8,9]$. Therefore, additional studies are required to gain insight into the exact correlation between WMHs and the features of migraine.

Larger or confluent WMHs are usually observed in cerebrovascular diseases and cognitive decline cases $[10$,

(c) The Author(s). 2018 Open Access This article is distributed under the terms of the Creative Commons Attribution 4.0 International License (http://creativecommons.org/licenses/by/4.0/), which permits unrestricted use, distribution, and 
11]. However, WMHs associated with migraine tend to be punctate and mild [10]. Nevertheless, studies that have investigated the clinical implication of WMHs are scarce. Longitudinal population-based studies have previously indicated that WMHs in migraine are not associated with stroke or the decline in cognitive function $[10,12]$. However, migraine has a variable short- or long-term prognosis. Some patients achieve complete or partial remission, while others experience persistent or even progressive attacks [13]. Recently, Eggers proposed that WMHs might be caused by multiple microemboli, which are induced by platelet aggregation abnormalities usually observed in migraine patients [14]. This supports the notion that WMHs may reflect an abnormal internal environment in patients. Therefore, the present study aimed to investigate the prognostic value of WMHs in migraine patients. To this end, the T1- and T2-weighted, as well as the T2-weighted FLAIR, magnetic resonance imaging (MRI) data obtained from migraine patients were analyzed to describe the imaging characteristics of WMHs. Furthermore, the association between WMHs and the clinical features of migraine were investigated, and the relationship between WMHs and migraine prognosis were examined.

\section{Methods}

\section{Patients}

A total of 69 migraine patients (52 females and 17 males, average age: 33.6 years old) were consecutively recruited from the Headache Clinic of the Department of Neurology, The First Affiliated Hospital of Xi'an Jiaotong University from February 2012 to November 2016. Inclusion criteria included: (1) patients with migraine who fulfilled the International Classification of Headache disorders (ICHD)-3 ( $\beta$ ) criteria [1]), and (2) age between 12 and 55 years old. Exclusion criteria: (1) patients with major neurological diseases, (2) patients with major systemic diseases, (3) patients with thyroid diseases, (4) pregnant and/or lactating patients, and (5) patients with claustrophobia. The present study was approved by the Ethics Committee of the First Affiliated Hospital of Xi'an Jiaotong University (XJTU1AF2015 LSK-159), and an informed written consent was obtained from each patient. For minor patients $(<16)$, the written consent form was obtained from the accompanying parents.

\section{Demographic characteristics and clinical features}

All enrolled patients were required to complete a standard questionnaire to collect basic clinical information at the Headache Clinic. This questionnaire assessed the demographic characteristics, past history, family history and features of migraine, and any accompanying symptoms and self-rating depression scale (SDS). Migraine features include disease duration, attack frequency, attack duration and the visual analogue scale (VAS) score. In addition, the questionnaire also investigated the history of smoking, hypertension, diabetes, oral contraceptive use, past vascular events, heart diseases, tumors, intracranial organic diseases and other medical conditions.

\section{Image data acquisition and evaluation of WMHs}

All participants underwent MRI scans at the Department of Radiology, The First Affiliated Hospital of Xian Jiaotong University, using a 3.0 T GE Discovery MR scanner and a standard 8-channel phase array head coil. High-resolution structural images were acquired using a three-dimensional T1-weighted sequence with the following parameters: TR, $10.276 \mathrm{~ms}$; TE, $4.9 \mathrm{~ms}$; matrix, $256 \times 256$; section-thickness, $1 \mathrm{~mm}$; FOV, $256 \mathrm{~mm}$. T2-weighted images were acquired with the following parameters: TR, $6000 \mathrm{~ms}$; TE, $104.4 \mathrm{~ms}$; matrix, $385 \times 384$; section-thickness, $5 \mathrm{~mm}$; FOV, $240 \mathrm{~mm}$. FLAIR images were acquired with the following parameters: TR, $9102 \mathrm{~ms}$; TE, $168.7 \mathrm{~ms}$; matrix, $288 \times 224$; section-thickness, $5 \mathrm{~mm}$; FOV, $240 \mathrm{~mm}$.

WMHs were visible as hyperintense lesions on FLAIR images, and as isointense or slightly hypointense lesions on T1-weighted images. MRI scans were assessed for the number and features of WMHs, including the appearance, number, size and anatomical location. All MRI scans were reviewed by an experienced neurologist and neuroradiologist. The degree of WMHs was assessed using the Scheltens visual rating scale [15]. Briefly, WMHs were separately graded in each of the following locations: frontal lobes, temporal lobes, parietal lobes and occipital lobes. WMHs were graded as follows: 0 (no lesions), 1 (hyperintensity $<3 \mathrm{~mm}$ and $n \leq 5$ ), 2 (hyperintensity $<3 \mathrm{~mm}$ and $n \geq 6$ ), 3 (hyperintensity 4 $10 \mathrm{~mm}$ and $n \leq 5$ ), 4 (hyperintensity $4-10 \mathrm{~mm}$ and $n \geq$ 6), 5 (hyperintensity $\geq 11 \mathrm{~mm}$ and $n \geq 1$ ), and 6 (confluent). The sum of scores from each location was considered as the final score $[15,16]$. According to $\mathrm{WMH}$, migraine patients were divided into two groups: non-WMH group (complete absence of WMHs or WMHs score $=0$ ) and WMH group (presence of WMHs or WMHs score $\geq 1$ ). Next, the features of WMHs and its correlation to the clinical variables were analyzed. Finally, a retrospective follow-up study was conducted to analyze the association of WMHs and migraine prognosis.

\section{Patient follow-up}

In December 2016, patients who were enrolled in the study for a mean period of $>24$ months $(n=45)$ were recruited for a follow up visit. A total of five patients were excluded due to percutaneous closure of the patent foramen ovale, and another seven patients dropped out from the follow up study. The study flow chart is illustrated in Fig. 1. 


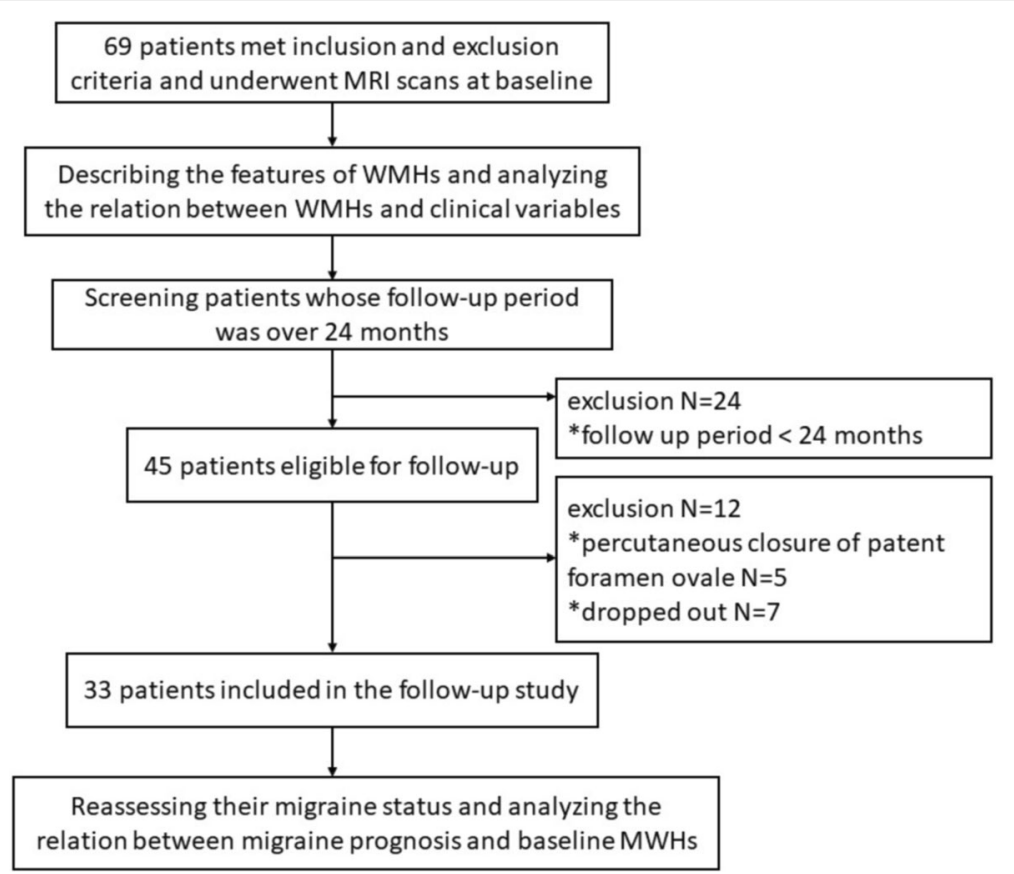

Fig. 1 A flow chart demonstrating the study design

A total of 33 patients were re-interviewed to assess their migraine status and determine the present headache attack frequency per month. Patients were divided into improved and non-improved groups based on the mean percentage of attack frequency calculated from the 3 months that preceded the follow-up appointment. The outcome was defined as improved if the attack frequency decreased by more than $50 \%$ compared to that of baseline. On the other hand, if the attack frequency failed to decrease by more than $50 \%$ at follow up, the patient was considered to be non-improved. Next, the outcome was further classified into four categories including complete remission, partial remission, persistence and progression. Complete remission was defined as zero migraine attacks in the 3 months that preceded the follow-up. Partial remission was defined as the reduction of migraine frequency by more than $50 \%$. Persistence group had the change of migraine frequency hovering around the $50 \%$. Progression was defined as the increase of migraine frequency by more than $50 \%$. It is worth mentioning that despite our efforts, most of the patients who were followed up denied to undergo a follow-up MRI scan.

\section{Therapeutic regimen}

The usage of medication during the follow-up period was recorded. Migraine patients were administered with migraine prophylactic medications (including calcium channel blockers, anticonvulsants, or $\beta$-blockers) or non-steroidal anti-inflammatory drugs, according to the recommendation of physicians.

\section{Statistical analysis}

Demographic and clinical characteristics were tabulated using descriptive statistics, including percentages, quartiles (non-normal data) and means (normal data). Chi-square test and Fisher's exact probability test were used to test for differences in categorical data. Student's $t$-test was used to compare the means of normally distributed variables. Non-parametric tests were used for non-normally distributed data. A correlation analysis between WMHs and patient prognosis was performed using Spearman correlation. Further, logistic regression was conducted to evaluate contributing factors to $\mathrm{mi}$ graine prognosis. Statistical analyses were performed using the SPSS 23.0 software. A $P$-value $<0.05$ was considered statistically significant.

\section{Results}

\section{Demographic characteristics and migraine features}

A total of 69 patients with an average age 33.6 years old (range: 14-54 years old) were enrolled in the present study. Among these patients, 52 patients $(75.4 \%)$ were females. The demographic characteristics and clinical features of migraine are summarized in Table 1. Furthermore, 19 patients $(27.5 \%)$ presented with aura including visual aura $(n=16)$, visual and sensory aura $(n=2)$, and brainstem aura $(n=1)$. Five patients were presented with chronic migraine, while the remaining patients were episodic. Moreover, a total of eight patients (11.6\%) were smokers, and another five patients (7.2\%) were hypertensive, but none of the enrolled patients were diabetic. 
Table 1 Comparison of clinical characteristics between the non-WMH group and WMH group

\begin{tabular}{|c|c|c|c|}
\hline & $\begin{array}{l}\text { Non-WMH } \\
\text { group }(n=45)\end{array}$ & $\begin{array}{l}\text { WMH group } \\
(n=24)\end{array}$ & $P$-value \\
\hline Age (year, mean $\pm S D$ ) & $30.6 \pm 10.4$ & $39.0 \pm 7.9$ & $<0.001^{*}$ \\
\hline Gender & & & 0.592 \\
\hline Female, $n(\%)$ & $33(73.3 \%)$ & $19(79.2 \%)$ & \\
\hline Male, $n(\%)$ & $12(26.7 \%)$ & $5(20.8 \%)$ & \\
\hline $\mathrm{BMI}\left(\mathrm{kg} / \mathrm{m}^{2}\right.$, mean $\left.\pm \mathrm{SD}\right)$ & $20.7 \pm 2.9$ & $21.8 \pm 2.2$ & 0.127 \\
\hline Hypertension, $n$ (\%) & $2(4.4 \%)$ & $3(12.5 \%)$ & 0.458 \\
\hline Smoking, $n(\%)$ & $5(11.1 \%)$ & $3(12.5 \%)$ & 1.000 \\
\hline Oral contraceptive use, n (\%) & $1(2.9 \%)$ & $1(5.3 \%)$ & 1.000 \\
\hline \multicolumn{4}{|l|}{ Headache characteristics } \\
\hline Aura, $n(\%)$ & $14(31.1 \%)$ & $5(20.8 \%)$ & 0.363 \\
\hline Disease duration (month, quartile) & $84.0(42.0,198.0)$ & $180.0(75.0,297.0)$ & $0.013^{*}$ \\
\hline Attack frequency (day/month, quartile) & $3.0(2.0,7.0)$ & $4.0(2.0,10.0)$ & 0.465 \\
\hline Attack duration (hour, quartile) & $5.0(3.0,10.0)$ & $9.5(4.0,24.0)$ & 0.172 \\
\hline Visual analogue scale, mean \pm SD & $7.0 \pm 1.9$ & $7.8 \pm 1.5$ & 0.080 \\
\hline \multicolumn{4}{|l|}{ Accompany symptoms } \\
\hline Nausea, $n(\%)$ & $37(84.1 \%)$ & $21(87.5 \%)$ & 0.983 \\
\hline Vomiting, $n(\%)$ & $28(63.6 \%)$ & $15(62.5 \%)$ & 0.926 \\
\hline Photophobia, n (\%) & $32(72.7 \%)$ & $18(75.0 \%)$ & 0.839 \\
\hline Phonophobia, n (\%) & $33(75.0 \%)$ & $18(75.0 \%)$ & 1.000 \\
\hline Dizziness, $n$ (\%) & $21(47.7 \%)$ & $13(54.2 \%)$ & 0.612 \\
\hline Family history of migraine, $n(\%)$ & $26(57.8 \%)$ & $11(45.8 \%)$ & 0.343 \\
\hline SDS scores (quartile) & $42(36,56)$ & $44.5(37.3,56.8)$ & 0.653 \\
\hline
\end{tabular}

${ }^{*}$ A significance level, $P<0.05$; SD standard deviation, $B M I$ body mass index

Next, WMHs were investigated through T2-weighted and FLAIR MRI scans. According to WMH, migraine patients were divided into two groups: non-WMH group (complete absence of WMHs or WMH score $=0$ ) and WMH group (the presence of WMHs or a WMH score of $\geq 1$ ) (Table 1). Among these 69 migraine patients, a total of 24 patients $(34.8 \%, 19$ females and five males) presented with WMHs. Furthermore, there was a significant difference in age between the WMH and non-WMH groups. Patients in the WMH group were significantly older compared to patients in the non-WMH group (39.0 \pm 7.9 years vs. $30.6 \pm 10.4$ years; $P=0.000$ ). Among the disease burden related variables, disease duration was significantly higher in the WMH group than in the non-WMH group (median: 180 months vs. 84 months; $P=0.013$ ). Furthermore, a moderate positive correlation was observed between age and disease duration $(r=0.589, P<$ 0.001 ), which indicate a possible confounding effect of age in the association between disease duration and WMHs. A scatter plot was preformed to show the changing trend of disease duration with age. The trend was similar between the improved group and non-improved group
(Fig. 2). No significant difference was observed in the presence of aura between these two groups. It is worth mentioning that the exclusion of hypertension or smoking status (12 cases) did not affect the data analyses between these two groups.

\section{Features of WMHs}

In the WMH group $(n=24)$, most lesions were punctuate (22 patients), and two patients presented with confluent lesions (Fig. 3b and c). Among patients with WMHs, a total of 171 lesions were detected. WMHs were significantly higher in the frontal lobes $(74.9 \%)$, followed by the parietal lobes (21.6\%) (Fig. 4a). Furthermore, it was observed that WMHs in migraine patients were generally mild, most lesions (94.7\%) were $<5 \mathrm{~mm}$ (Fig. $4 \mathrm{~b}$ ), and the average number of lesions per patient was generally small, with a median number of 2.5 (range: 1-52) (Fig. 4c). According to the Scheltens scale, the WMH scores of patients were low, with a median score of 2.5 (range: $0-10$ ) (Fig. 4d). Furthermore, the difference in numbers and scores between males and females with WMHs was not statistically significant. 


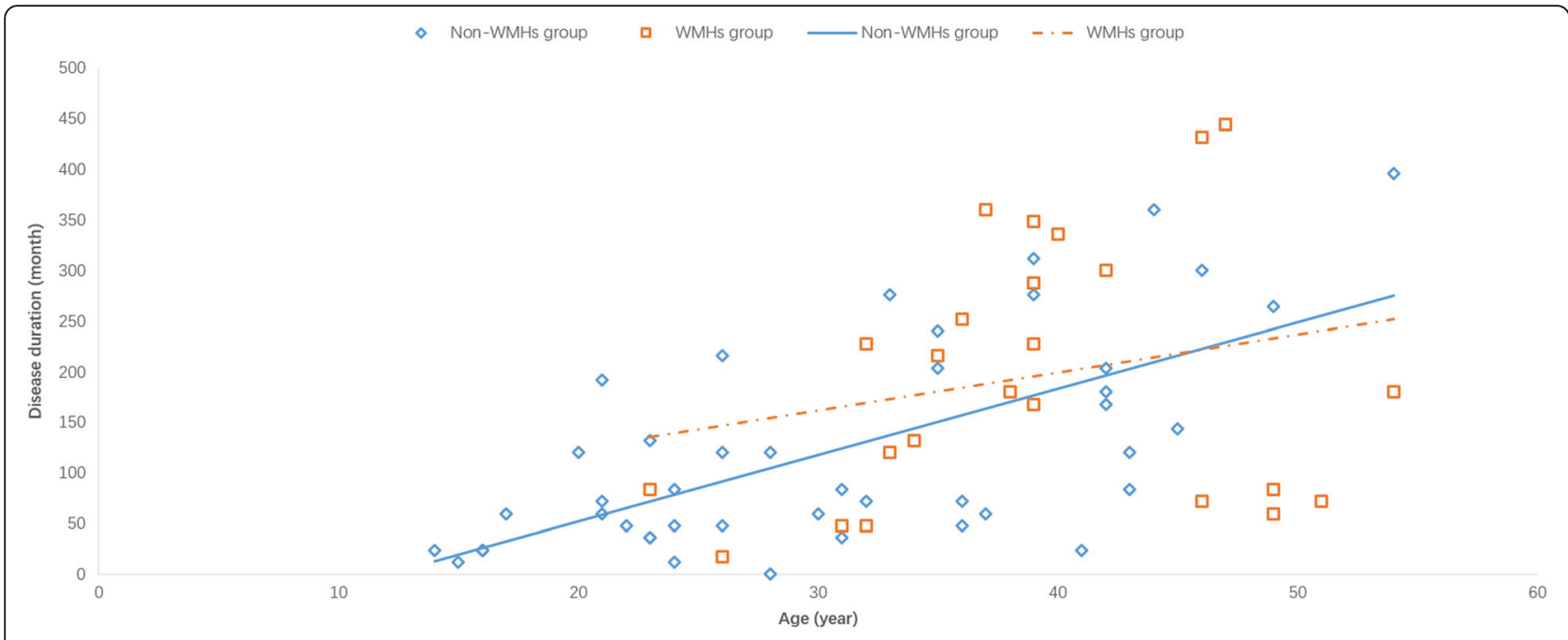

Fig. 2 A scatter plot showing the changing trend of disease duration with age

\section{Correlation between WMHs and migraine prognosis}

A total of 33 patients were followed up in the present study (Fig. 1). The average follow-up period was 3 years, which ranged within 2-4 years. Among these 33 re-assessed patients, 15 patients (45.5\%) were assigned to the improved group while 18 patients (54.5\%) were assigned to the non-improved group. Specifically, 4 patients (12.1\%) achieved complete remission and they were free of migraine attacks for more than 1 year. Eleven patients $(33.3 \%)$ achieved partial remission. Fourteen patients $(42.4 \%)$ were in persistence group. Four patients $(12.1 \%)$ were in the progression group. The four patients attaining complete remission were $30,34,39$ and 41 years old at follow-up, excluding the potential effect of menopausal state. Differences in age, gender, or BMI between the improved and non-improved groups were not statistically significant. None of our patients had hypertension or diabetes. Among the headache characteristics, aura was more frequent in the improved group $(60.0 \%$ vs. $11.1 \%, P=0.008)$ (Table 2$)$.

Among the 18 patients in the non-improved group, 10 patients had one or more WMHs, while WMHs were detected in only two of 15 patients in the improved group, and the difference was statistically significant $(P=0.027$, Table 3). Furthermore, patients in the non-improved group had a significantly higher median WMHs score compared to patients in the improved group $(P=0.030)$.

Next, we examined the impact of prophylactic treatments on the migraine outcome. We compared the rate of regular prophylactic treatment between the improved and non-improved groups administered for more than 3 months regardless of drug classes or doses. Our results demonstrated the absence of significant difference between both patient groups (Table 2). It is worth mentioning that none of the patients reported the use triptans.

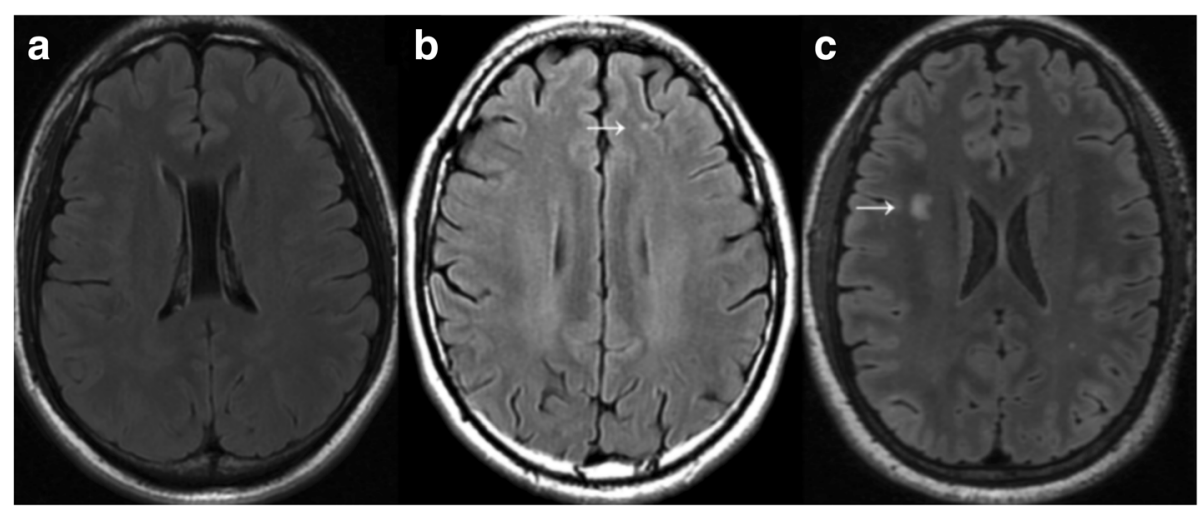

Fig. 3 Representative axial FLAIR images of WMHs: (a) Normal brain structures without white matter hyperintensity. b A punctate hyperintense lesion (arrow) in the right frontal lobe. c A confluent lesion (arrow) and some punctate lesions in the brain 


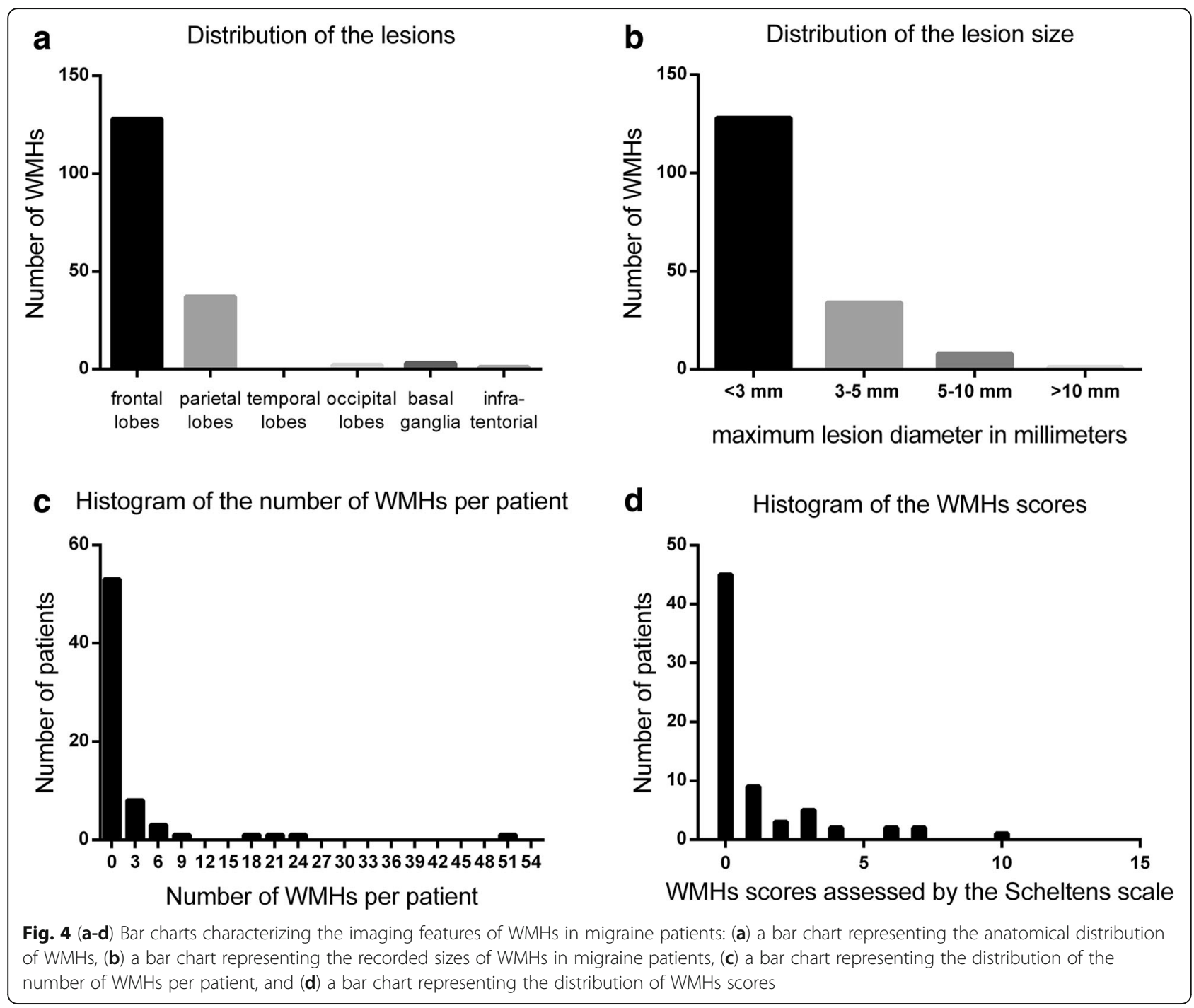

Multivariate logistic regression analysis was conducted to analyze the independent risk factor associated with the non-improved outcome. The impact of age, aura, disease duration, VAS and the presence of WMHs were examined. Our results showed that WMHs $(\mathrm{OR}=12.6,95 \% \mathrm{CI}(1.093 \sim 145.848)$ and aura $(\mathrm{OR}=0.04,95 \% \mathrm{CI}(0.002 \sim 0.683)$ were the independent risk factors associated with the non-improved outcome.

In the follow up group, patients without aura were significantly older than those with aura $(36.6 \pm 9.1$ vs. $23.8 \pm 8.3, P<0.001)$ and had a significantly longer disease duration (median: 162 months vs. 36 months, $P=0.003$ ) and attack duration (median: $2 \mathrm{~h}$ vs. $11 \mathrm{~h}, \mathrm{P}<0.001$ ). Patients without aura also had a higher incidence of WMHs than those with aura ( $45.4 \%$ vs. $18.2 \%, P=0.249)$. The difference was obvious although it did not achieve the statistical significance.

\section{Discussion}

Migraine is a well-documented risk factor for WMHs [3, 5, $17,18]$. However, to date, the clinical significance of WMHs in migraine prognosis remains unclear. Therefore, in the present study, we explored the association between WMHs and migraine prognosis. Results demonstrated that the presence and degree of WMHs can be associated with unfavorable migraine prognosis. In addition, in the present study, we described the features of WMHs in migraine patients, and observed that WMHs were positively correlated with old age.

WMHs are commonly associated with physiological conditions such as aging and pathological conditions associated with vascular risks such as hypertension [19]. Pathologically, WMHs can result from local brain ischemia at the microvascular level $[14,19]$. Several reports have demonstrated a higher incidence of WMHs in patients with migraine, compared to healthy control subjects 
Table 2 Comparison of demographics and WMHs between the improved and non-improved groups

\begin{tabular}{|c|c|c|c|}
\hline & $\begin{array}{l}\text { Improved } \\
\text { group }(n=15)\end{array}$ & $\begin{array}{l}\text { Non-improved } \\
\text { group }(n=18)\end{array}$ & $P$-value \\
\hline Age (year, mean $\pm S D)$ & $29.5 \pm 9.8$ & $34.7 \pm 11.0$ & 0.166 \\
\hline Gender & & & 1.000 \\
\hline Female, $n(\%)$ & $13(86.7 \%)$ & $16(88.9 \%)$ & \\
\hline Male, $n(\%)$ & $2(13.3 \%)$ & $2(11.1 \%)$ & \\
\hline $\mathrm{BMI}\left(\mathrm{kg} / \mathrm{m}^{2}\right.$, mean $\left.\pm \mathrm{SD}\right)$ & $19.9 \pm 2.3$ & $21.1 \pm 2.9$ & 0.203 \\
\hline \multicolumn{4}{|l|}{ Headache characteristics } \\
\hline Aura, $n(\%)$ & $9(60.0 \%)$ & $2(11.1 \%)$ & $0.008^{*}$ \\
\hline Disease duration (month, quartile) & $72(24,144)$ & $150(48,279)$ & 0.096 \\
\hline Attack frequency (day/month, quartile) & $4.0(2.0,7.0)$ & $2.0(2.0,5.5)$ & 0.555 \\
\hline Attack duration (hour, quartile) & $4.5(2.0,10.0)$ & $11.0(3.4,24.0)$ & 0.204 \\
\hline Visual analogue scale, mean \pm SD & $7.0(6.0,7.9)$ & $8.0(7.0,9.3)$ & 0.051 \\
\hline \multicolumn{4}{|l|}{ Medications } \\
\hline Prophylactic medications ${ }^{\#}, n(\%)$ & $5(33.3 \%)$ & $4(22.2 \%)$ & 0.697 \\
\hline Medication overuse $e^{\#}, n(\%)$ & $1(6.7 \%)$ & $2(11.1 \%)$ & 1.000 \\
\hline
\end{tabular}

${ }^{*}$ A significance level, $P<0.05 ;{ }^{\#}$ The number of patients who took regular prophylactic medications for more than 3 months or had medication overuse during the follow-up period; SD standard deviation, $B M I$ body mass index

$[3,5]$. In the same context, Schurks et al. demonstrated that migraine is a definite risk of stroke, especially in young women [20]. This suggests the role of ischemia in the mechanism of WMHs in association with migraine. To date, the exact pathophysiology of WMHs is not well-understood. Accumulating evidence revealed that migraine patients may have abnormal platelet activation, impaired endothelial function and hypercoagulability [21-23], which can be potential causes for the development of WMHs. These abnormal vascular conditions might favor the persistence or even the progression of migraine. Consequently, it is reasonable to speculate that WMHs can be correlated with unfavorable migraine prognosis. Indeed, results obtained from the present study demonstrate that both the degree and frequency of WMHs were positively correlated with unfavorable migraine prognosis. To the best of our knowledge, this is the first report that demonstrated the prognostic value of WMHs in migraine patients.

Aging is another important risk factor of the development of WMHs $[4,19]$. Among the examined clinical variables, only age and disease duration were correlated to WMHs in our patient cohort. However, a moderate positive correlation was observed between age and disease

Table 3 Fisher's exact test representing the correlation between WMHs and patient prognosis

\begin{tabular}{llll}
\hline & Improved & Non-improved & Total \\
\hline WMHs group & 2 & 10 & 12 \\
Non-WMHs group & 13 & 8 & 21 \\
Total & 15 & 18 & 33
\end{tabular}

Fisher's exact test: two-tailed $P$-value $=0.027$ duration. The disease duration of patients with WMHs was not higher than that of patients without WMHs within the same age brackets. These results imply that the association between disease duration and WMHs could result from the confounding effect of age. Furthermore, we investigated whether age had a confounding effect on the association of WMHs and migraine prognosis. There was no significant difference in age between the improved and non-improved groups. Regression analysis showed WMHs was the independent risk factor for the non-improved outcome with the control of the cofounding effect of age. Finally, the rate of prophylactic treatment was also comparable between these two groups. Collectively, these results indicate that the association between WMHs and migraine prognosis were not affected by age or medications in our patient cohort. However, it is recognized that the prevalence of migraine increases with age from childhood to adulthood, and it peaks at 35 to 39 years of age, after which it gradually decreases, particularly among women after menopause [3]. Meanwhile, WMHs are not static, and in most cases WMHs progress with aging [24, 25]. Therefore, for older migraine patients, WMHs will not serve as a reliable marker for prognosis. It is worth mentioning that a 3-year longitudinal follow-up study revealed a non-significant increase in the number of WMHs in $19.5 \%$ of the patient cohort [26]. These results suggest that WMHs would not significantly progress within a relatively short period (3 years). In the present study, patients were re-evaluated after 2-4 years (mean: 3 years). Therefore, this might indicate that the validity of WMHs in 
migraine prognosis is at least applicable over a relatively short interval (3-year window). Future research should examine its validity through long-term follow ups.

In this study, our results demonstrated that migraineurs with favorable outcome had a higher incidence of aura. Studies analyzing the relationship between the presence of aura and migraine prognosis are scarce. Dahlof et al. observed that aura was associated with poor migraine prognosis in females, but a similar relationship was not observed in male patients [27]. A 5-year follow-up study that investigated the outcome of migraine in children and adolescents failed to find a significant difference between migraine with and without aura, although the percentage of subjects who were free from migraine at follow-up was $30.6 \%$ in the case of migraine with aura and $20.3 \%$ in the case of migraine without aura [28]. In our study cohort, patients with aura were significantly younger than those without aura. Disease duration and attack duration were significantly lower in patients with aura than those without aura. Patients with aura had a lower incidence of WMHs than those without aura although the difference was not significant. On the other hand, Gozke et al. previously suggested a higher incidence of WMHs in migraine with aura [29]. Moreover, it is well recognized that migraine with aura is a risk associated with ischemic stroke [20]. Therefore, it is plausible to speculate that the better baseline headache condition in patients with aura could contribute to better prognosis compared to patients without aura. However, the exact reason remains to be clarified in future studies.

There was no significant difference of SDS scores between WMHs group and Non-WMHs group. The relationship between depression and WMHs remains unclear [30, 31]. A meta-analysis showed a significant weak association between WMHs and depression (OR: 1.02 1.22) [32]. The burden of migraine can be assessed by disease duration, attack frequency, attack duration and headache intensity (VAS) [18]. In the present study, we investigated the impact of WMHs on disease burden. It was observed that WMHs were significantly associated with longer disease duration, while a significant correlation with attack frequency or its duration was not observed. To date, the association between WMHs and migraine features remain controversial $[6,18,33,34]$. Earlier reports have consistently revealed that WMHs were not associated with migraine features, including disease duration or attack duration [35-37]. On the other hand, Gozke et al. demonstrated that WMHs were associated with a higher frequency of longer disease duration and higher attack frequencies [29]. Similarly, the CAMERA study supported the same conclusions [3]. However, the population of the CAMERA study had high proportions of vascular risk factors (32-42\% prevalence of hypertension, 60-66\% prevalence of smoking, and $19-29 \%$ prevalence of oral contraceptive use), which may lead to confounding bias. This discrepancy might be attributed to the accuracy of the MRI techniques, especially the blurring artifact. Other factors include the demographic characteristics of the study population or discrepancies in the study design $[3,6,29$, $34,35]$. In addition, the measurements of disease burden are usually not stable; that is, disease duration was always affected by age, and attack frequency and attack duration often show changeable patterns over time; while the VAS had strong subjectivity. Therefore, future research should focus on more stable parameters to assess the disease burden of migraine.

Taken together, the results obtained from the study suggest that WMHs may predict unfavorable migraine prognosis. Therefore, our results could lead to the alteration of the treatment protocol for migraineurs with WMHs. That is, physicians could apply more positive treatment strategies to achieve a more favorable prognosis in patients with high WMHs scores. Furthermore, our results also indicate that WMHs have a closer association with age than the clinical features of migraine.

Nevertheless, the present study had a few limitations. The relatively small number of enrolled patients is considered to be the main limitation. Furthermore, the absence of a control group precluded definitive conclusions about the nature of the observed alterations in WMHs or whether their degree is beyond normal aging. Age should be controlled in the design of the study. Thus, future work should focus on investigating the implication of WMHs among relatively young migraine patients. Similarly, the heterogeneity of the patient cohort such as migraine with and without aura, episodic migraine and chronic migraine, should be improved. Different migraine types possibly have different effects on the prognosis. The relatively low migraine frequency at baseline in our study is also a major limitation as prognostic information may be of greater value in high frequency migraine states. However, the small sample size limited the stratified analysis of frequency in the case of controlling the effect of age. The method for migraine prognosis categorization was one-sided. It reflected the change of frequency but it did not investigate the current frequency level, headache intensity or even response to acute therapy. However, there is no standard prognostic categorization for migraine yet. Future prospective multicenter studies with more controlled conditions (migraine type and age) and long-term follow up should be conducted to confirm these results. Future studies should also employ more stable parameters that assess disease burden, in order to further confirm the clinical significance of WMHs. It is worth mentioning that in the present study, we could not definitively investigate other 
WMH-associated risk factors, including hypertension, diabetes, hyperlipidemia, hyperhomocysteinemia, hyperuricemia, hypercoagulability, heart diseases, kidney diseases, inflammation and autoimmune diseases. These conditions may impact the strength of our conclusions regarding the nature of WMHs and their effect on migraine. Future studies should be controlled for the confounding effects of the above mentioned conditions.

\section{Conclusion}

This study suggests that WMHs can predict short-term unfavorable migraine prognosis, thereby providing a new insight into the clinical significance of WMHs in migraine. Meanwhile, it demonstrates that WMHs in migraine patients are generally mild, mostly located in the frontal and parietal lobes, and may have a closer association with age than headache characteristics.

\section{Abbreviations}

FLAIR: Fluid-attenuated inversion recovery; VAS: Visual analogue scale; WMHs: White matter hyperintensities

\section{Funding}

This work was supported by the National Key Technology R\&D Program of China (No. 2014BAI04B05) and the National Natural Science Foundation of China (No. 81271127).

\section{Availability of data and materials}

The datasets used and/or analyzed during the current study are available from the corresponding author upon request.

\section{Authors' contributions}

GGL, HX, KH and JY designed the study. HX, QZ, RL, ZJJ, YTB, DZ and LHZ collected and analyzed the data. HX, QZ and $\mathrm{KH}$ drafted and wrote the manuscript. HX, GGL, RL, ZJJ, DZ, LHZ, GLL and JY revised the manuscript critically for intellectual content. All authors provided intellectual input to the study and approved the final version of the manuscript.

\section{Ethics approval and consent to participate}

The present study was approved by the Ethics Committee of the First Affiliated Hospital of Xi'an Jiaotong University (XJTU1AF2015 LSK-159). All procedures performed in studies involving human participants were in accordance with the ethical standards of the institutional and national research committee, and the 1964 Helsinki declaration and its later amendments or comparable ethical standards. A written informed consent was obtained from all individual participants included in the study. For minor patients $(<16)$, the written consent form was obtained from the accompanying parents.

\section{Consent for publication}

A written consent form was obtained from all participants for potentially publishing their clinical data and images while protecting their personal information. For participants under the age of 16, we obtained the written consent form from their parents.

\section{Competing interests}

The authors declare that they have no competing interests.

\section{Publisher's Note}

Springer Nature remains neutral with regard to jurisdictional claims in published maps and institutional affiliations.

\section{Author details}

'Department of Neurology, The First Affiliated Hospital of Xi'an Jiaotong University, No. 277 Yanta West Road, Xi'an 710061, Shaanxi, China. ${ }^{2}$ Department of Neurology, Shaanxi Provincial People's Hospital, Xi'an
710068, China. ${ }^{3}$ Department of Radiology, The First Affiliated Hospital of Xi'an Jiaotong University, Xi'an 710061, China. ${ }^{4}$ Arrhythmia Unit, Department of Cardiovascular Medicine, The First Affiliated Hospital of Xi'an Jiaotong University, Xi'an 710061, China.

Received: 21 August 2017 Accepted: 25 June 2018

Published online: 02 July 2018

\section{References}

1. Headache Classification Committee of the International Headache S. The international classification of headache disorders, 3rd edition (beta version). Cephalalgia. 2013;33:629-808.

2. Steiner TJ, Stovner LJ, Birbeck GL. Migraine: the seventh disabler. J Headache Pain. 2013;14:1.

3. Kruit MC, van Buchem MA, Hofman PA, Bakkers JT, Terwindt GM, Ferrari MD, et al. Migraine as a risk factor for subclinical brain lesions. JAMA. 2004;291: 427-34.

4. Lin J, Wang D, Lan L, Fan Y. Multiple factors involved in the pathogenesis of white matter lesions. Biomed Res Int. 2017;2017:9372050.

5. Swartz RH, Kern RZ. Migraine is associated with magnetic resonance imaging white matter abnormalities: a meta-analysis. Arch Neurol. 2004;61:1366-8.

6. Trauninger A, Leel-Ossy E, Kamson DO, Poto L, Aradi M, Kover F, et al. Risk factors of migraine-related brain white matter hyperintensities: an investigation of 186 patients. J Headache Pain. 2011:12:97-103.

7. Toghae M, Rahimian E, Abdollahi M, Shoar S, Naderan M. The prevalence of magnetic resonance imaging Hyperintensity in migraine patients and its association with migraine headache characteristics and cardiovascular risk factors. Oman Med J. 2015;30:203-7.

8. Zhang Q, Datta R, Detre JA, Cucchiara B. White matter lesion burden in migraine with aura may be associated with reduced cerebral blood flow. Cephalalgia. 2016;

9. Uggetti C, Squarza S, Longaretti F, Galli A, Di Fiore P, Reganati PF, et al. Migraine with aura and white matter lesions: an MRI study. Neurol Sci. 2017; 38:11-3.

10. Friedman DI, Dodick DW. White matter hyperintensities in migraine: reason for optimism. JAMA. 2012;308:1920-1.

11. Dufouil C, Godin O, Chalmers J, Coskun O, MacMahon S, Tzourio-Mazoyer N, et al. Severe cerebral white matter hyperintensities predict severe cognitive decline in patients with cerebrovascular disease history. Stroke. 2009:40: 2219-21.

12. Kurth T, Mohamed S, Maillard P, Zhu YC, Chabriat H, Mazoyer B, et al. Headache, migraine, and structural brain lesions and function: population based epidemiology of vascular ageing-MRI study. BMJ. 2011;342:C7357.

13. Bigal ME, Lipton RB. The prognosis of migraine. Curr Opin Neurol. 2008;21: 301-8.

14. Eggers AE. Migraine white matter hyperintensities and cerebral microinfarcts are silent cryptogenic strokes and relate to dementia. Med Hypotheses. 2017;102:1-3.

15. Scheltens P, Barkhof F, Leys D, Pruvo JP, Nauta JJP, Vermersch P, et al. A semiquantitative rating scale for the assessment of signal hyperintensities on magnetic resonance imaging. J Neurol Sci. 1993;114:7-12.

16. Adami A, Rossato G, Cerini R, Thijs VN, Pozzi-Mucelli R, Anzola GP, et al. Right-to-left shunt does not increase white matter lesion load in migraine with aura patients. Neurology. 2008;71:101-7.

17. Kruit MC, van Buchem MA, Launer $\sqcup$, Terwindt GM, Ferrari MD. Migraine is associated with an increased risk of deep white matter lesions, subclinical posterior circulation infarcts and brain iron accumulation: the populationbased MRI CAMERA study. Cephalalgia. 2010;30:129-36.

18. Yilmaz Avci A, Lakadamyali H, Arikan S, Benli US, Kilinc M. High sensitivity Creactive protein and cerebral white matter hyperintensities on magnetic resonance imaging in migraine patients. J Headache Pain. 2015;16:9.

19. Pantoni L. Cerebral small vessel disease: from pathogenesis and clinical characteristics to therapeutic challenges. Lancet Neurol. 2010;9:689-701.

20. Schurks M, Rist PM, Bigal ME, Buring JE, Lipton RB, Kurth T. Migraine and cardiovascular disease: systematic review and meta-analysis. Br Med J. 2009;339

21. Tietjen GE, Collins SA. Hypercoagulability and migraine. Headache. 2018:58: 173-83.

22. Danese $\mathrm{E}$, Montagnana M, Lippi G. Platelets and migraine. Thromb Res. 2014;134:17-22.

23. Tietjen GE, Khubchandani J. Vascular biomarkers in migraine. Cephalalgia. 2015:35:95-117. 
24. Palm-Meinders $\mid \mathrm{H}$, Koppen $\mathrm{H}$, Terwindt $\mathrm{GM}$, Launer $L$, Konishi J, Moonen JM, et al. Structural brain changes in migraine. JAMA. 2012;308:1889-97.

25. Rozen TD. Images from headache: white matter lesions of migraine are not static. Headache. 2010;50:305-6.

26. Dinia L, Bonzano L, Albano B, Finocchi C, Del Sette M, Saitta L, et al. White matter lesions progression in migraine with aura: a clinical and MRI longitudinal study. J Neuroimaging. 2013;23:47-52.

27. Dahlof CG, Johansson M, Casserstedt S, Motallebzadeh T. The course of frequent episodic migraine in a large headache clinic population: a 12-year retrospective follow-up study. Headache. 2009:49:1144-52.

28. Cuvellier JC, Tourte M, Lucas C, Vallee L. Stability of pediatric migraine subtype after a 5-year follow-up. J Child Neurol. 2016;31:1138-42.

29. Gozke E, Ore O, Dortcan N, Unal Z, Cetinkaya M. Cranial magnetic resonance imaging findings in patients with migraine. Headache. 2004; 44:166-9.

30. Versluis $C E$, van der Mast $R C$, van Buchem MA, Bollen EL, Blauw GJ, Eekhof JA, et al. Progression of cerebral white matter lesions is not associated with development of depressive symptoms in elderly subjects at risk of cardiovascular disease: the PROSPER study. Int J Geriatr Psychiatry. 2006;21:375-81.

31. Firbank MJ, O'Brien JT, Pakrasi S, Pantoni L, Simoni M, Erkinjuntti T, et al. White matter hyperintensities and depression-preliminary results from the LADIS study. Int J Geriatr Psychiatry. 2005;20:674-9.

32. Wang L, Leonards CO, Sterzer P, Ebinger M. White matter lesions and depression: a systematic review and meta-analysis. J Psychiatr Res. 2014; 56:56-64.

33. Gaist D, Garde E, Blaabjerg M, Nielsen HH, Kroigard T, Ostergaard K, et al. Migraine with aura and risk of silent brain infarcts and white matter hyperintensities: an MRI study. Brain. 2016;139:2015-23.

34. Galli A, Di Fiore P, D'Arrigo G, Uggetti C, Squarza S, Leone M, et al. Migraine with aura white matter lesions: preliminary data on clinical aspects. Neurol Sci. 2017;38:7-10.

35. Pavese N, Canapicchi R, Nuti A, Bibbiani F, Lucetti C, Collavoli P, et al. White matter MRI hyperintensities in a hundred and twenty-nine consecutive migraine patients. Cephalalgia. 1994;14:342-5.

36. De Benedittis G, Lorenzetti A, Sina C, Bernasconi V. Magnetic resonance imaging in migraine and tension-type headache. Headache. 1995;35:264-8.

37. Cooney BS, Grossman RI, Farber RE, Goin JE, Galetta SL. Frequency of magnetic resonance imaging abnormalities in patients with migraine. Headache. 1996;36:616-21.

\section{Ready to submit your research? Choose BMC and benefit from:}

- fast, convenient online submission

- thorough peer review by experienced researchers in your field

- rapid publication on acceptance

- support for research data, including large and complex data types

- gold Open Access which fosters wider collaboration and increased citations

- maximum visibility for your research: over $100 \mathrm{M}$ website views per year

At BMC, research is always in progress.

Learn more biomedcentral.com/submissions 